\title{
Simulex 3.0: Modelling Evacuation in Multi-Storey Buildings
}

\author{
PETER THOMPSON \\ Integrated Environmental Solutions Ltd. \\ 1, Atlantic Quay, Broomielaw, Glasgow G2 8JE, UK \\ JIANHUA WU \\ State Key Laboratory of Fire Science \\ University of Science and Technology of China \\ Hefei, Anhui 230026, P.R. China \\ ERIC MARCHANT \\ Fire Safety Engineering Group \\ University of Edinburgh \\ Department of Civil and Environmental Engineering \\ Kings Buildings \\ Edinburgh EH9 3JN, UK
}

\begin{abstract}
This paper describes the further development of the evacuation software 'Simulex'. The aim of the research project is to develop a computer model for evacuation which has a strong scientific basis for the simulated movement of escaping people, and can be readily used as a research and design tool. The software has been written by researchers at Edinburgh, in collaboration with staff at Lund University. The project has been funded by The Swedish Fire Research (Brandforsk) board and The Engineering and Physical Sciences Research Council.

The software is capable of modelling large, geometrically complex buildings, with multiple floors and staircases, and accepts CAD generated files to define individual floor plans. Thousands of individual people can be accommodated, and the user can view the movement of each individual at any point in the building at any time during the evacuation. A text file which contains detailed information about the evacuation process is produced at the end of a simulation. These capabilities represent significant advancements in the software described previously by Thompson \& Marchant [8].
\end{abstract}

\section{KEYWORDS}

Evacuation, simulation, speeds, distance maps, route-finding, overtaking, side-stepping, human response, behaviour, validation. 


\section{SOFTWARE DESCRIPTION}

Simulex runs on any 486 / Pentium based PC, with Windows v3.1/95/NT and at least $8 \mathrm{Mb}$ RAM installed. It runs entirely under Windows as a single package, and all graphics and menus are handled through the Windows interface. The software is written in Microsoft Visual $\mathrm{C}++1.51$ for Windows.

\section{INTRODUCTION}

Simulex was originally developed as part of a $\mathrm{PhD}$ thesis by Thompson [10]. The second version was a DOS-based program, which only accommodated evacuation movement on a single floor plan, was very limited in terms of psychological modelling, and used only one distance 'route' map where each person always travelled towards the nearest exit. Simulex 3.0 represents significant improvements in all of these areas, in addition to providing full Windows compatibility and the capability to access CAD generated files to define floor plans.

\section{DEFINING A MULTI-STOREY BUILDING}

Each floor plan and each staircase in a building is displayed in a different viewing 'window'. Simulex defines a multi-storey building as a series of 2-dimensional floor plans which are connected by staircases. The exit openings which lead from each floor into a staircase are fixed in both the floor plan 'window' and the staircase 'window'. The staircases and floor plans are connected by links which are placed at the exit openings. These links are used later to allow the modelled occupants to step from a floor plan into a staircase and vice-versa.

The following inputs are required from the user:

- FLOOR PLANS: A plan of each floor of the building is required by Simulex. The floor plan must have been previously defined with a CAD package, such as AutoCAD, CADD, or QuickCAD. The plan drawing must be saved in the standard 2-dimensional DXF file format. The user enters Simulex and loads the DXF file for each floor plan, and assigns a descriptive name to the defined floor level. When a DXF drawing is imported, the only information that is read by Simulex is that which defines the exact outlines of all walls and building fixtures. Dimensioning lines, notation, and door symbols are ignored as long as they have been defined on a different $\mathrm{CAD}$ layer (standard CAD drawing construction).

- STAIRCASES: Staircases are not defined with CAD drawings, but are instead defined within Simulex. In order to simplify user-input and to facilitate an easy viewing point for the user, the three-dimensional 'spiral' geometry of a typical staircase in a building is reduced to a linear two-dimensional form. Simulex assumes that a staircase can be represented by a linear 2-D 'corridor' within which each person's walking speed is reduced in comparison with walking in an open, horizontal floor space. The length of this 'stair- 
corridor' is equal to the mean length of the three-dimensional staircase. While this conversion of a staircase from a 3-D to a 2-D form leads to some loss in geometric accuracy, the gains in ease of user-input and viewing are significant. This two-dimensional conversion is required in order that automated route-finding algorithms (described later) can be applied.

- LINKS: The user is required to define 'links' of specified width between floor plans and staircases, in order to model the general three-dimensional form of a multi-storey building. A link is required whenever there is an opening between a floor plan and staircase. Figure 1 illustrates a simple building consisting of 2 floor plans (with similar layouts) and a staircase. The two doorways between the floor plans and staircase are represented by Links 1 and 2, which each possess a defined width and position. In Figure 1, the links are shown as ' $T$ ' shaped markers. Each link is displayed in both the floor and staircase windows. The 'links' can easily be moved and rotated in the displayed window by using the 'mouse'.

- LINES OF FINAL EXIT: A line of 'final exit' represents the final destination of occupants in a building

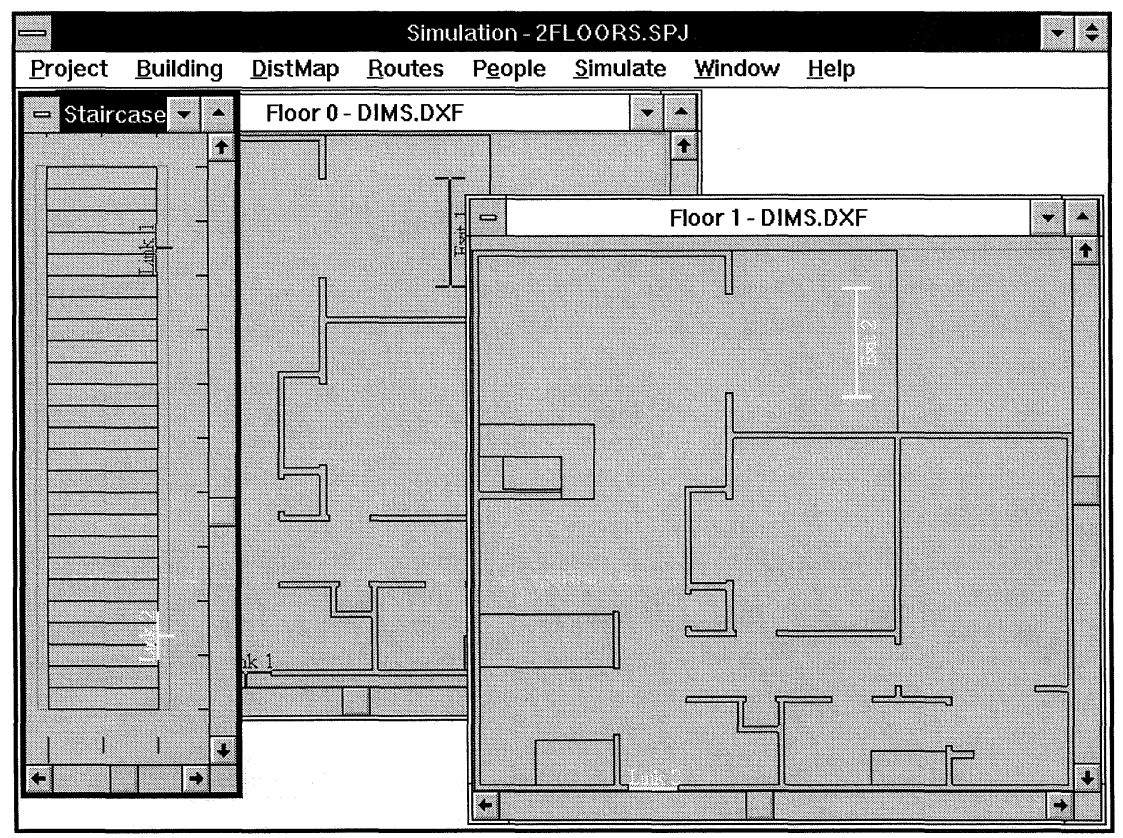

Figure 1. Sample Screen Display of a Simple Two-Storey Building. 
When an occupant reaches a line of 'final exit' he/she is removed from the building and is deemed to have escaped. Many of these lines can be defined near or beyond the boundaries of a building space, and two are used in the example shown in Figure 1. The large 'I' shapes represent lines of 'final exit'. The 'lines of final exit' each possess a specified width and orientation, and can easily be moved and rotated in the viewing window by using the 'mouse'.

\section{DISTANCE-MAPPING}

When a the building geometry has been defined, as described above, then Simulex is able to analyse the building space. Each floor plan and staircase is segmented into a grid of $0.2 \times 0.2 \mathrm{~m}$ square blocks, with each block assigned a numerical value in the computer's memory. An algorithm which calculates the distance to the nearest exit from any $0.2 \times 0.2 \mathrm{~m}$ block in the building, is applied to the linked, segmented, building space. The subsequent output is known as a 'distance map', which is illustrated in Figure 2. The values of distance-to-nearest-exit are represented by shaded bands where the 'colour' shading is graduated to represent contours of $1 \mathrm{~m}$ distance.

Distance maps are very useful when assessing travel distances in a building, and are also used by the route-finding functions during a simulated evacuation.

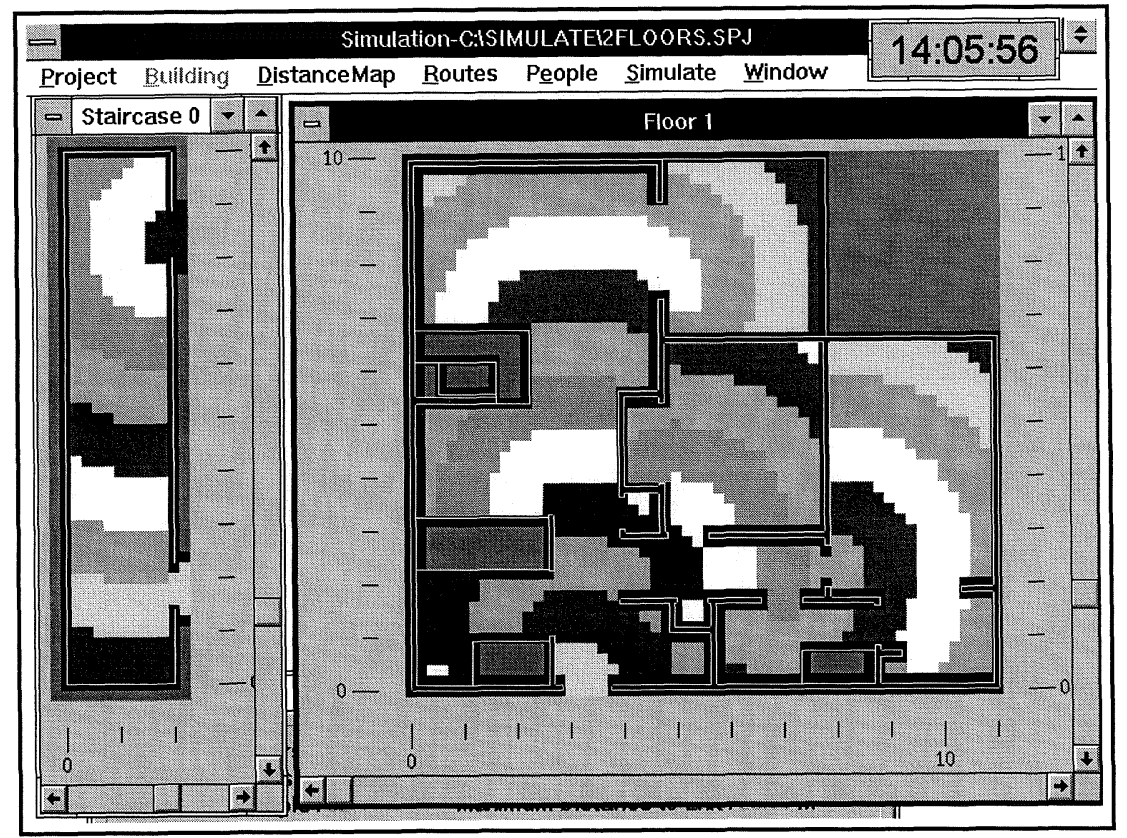

Figure 2. Sample Distance Map (with $1 \mathrm{~m}$ contours) Showing a Single Floor and Staircase. 
Simulex 3.0 allows the user to define more than 1 distance map for any building. Different distance maps may use different 'lines of final exit'. For example, the user may first create a distance map where all exits are recognised, and then also create an additional distance map where only those exits that are normally used by the occupants are incorporated. Furniture can also be drawn into the source $\mathrm{CAD}$ drawing file, which enables the distance map to analyse the effect of large obstructions on the escape paths.

Up to 10 different distance maps can be created and used, to enable a choice of different escape routes through the building space.

\section{THE BUILDING POPULATION}

The building population can be defined one person at a time by the user, or as large groups, spread over specified areas of the building. Groups are defined by clicking onto a series of points to create a polygonal area within a floor / staircase, and then using the option to create or delete a group of people within that area. If the user wishes to create a group of people, then the specified number of people will be placed within the defined area at regular spacing intervals. Alternatively, the user can place a single person at any point in the building, by clicking onto any position in the building space.

The characteristics that are assigned to a single individual or to individuals within a defined group, are governed by the options specified in the 'person characteristics' options box illustrated in Figure 3. Properties such as body type (controlling body size, and unimpeded walking speed), chosen distance map, and response time are defined by the user.

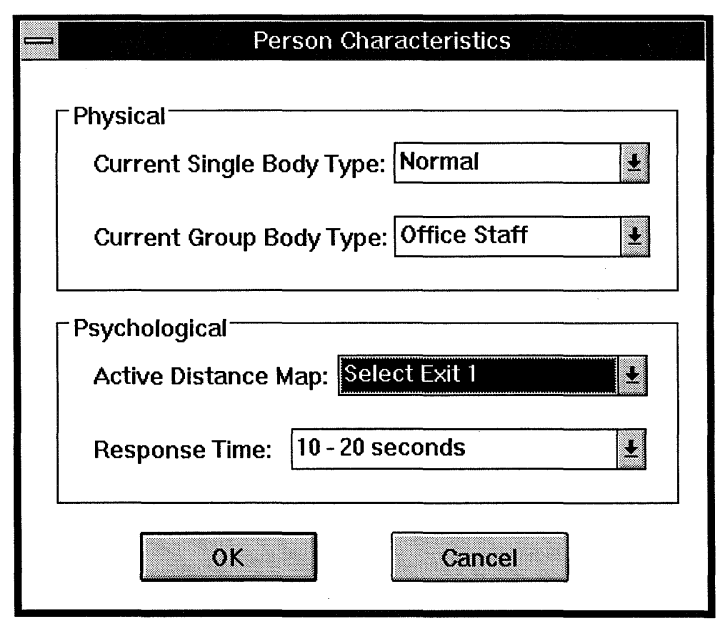

Figure 3. Sample Window for the Definition of Person Characteristics. 
The options selected as 'Person Characteristics' either define fixed parameters, such as which distance map to use (route-to-exit), or distributed parameters such as response time and body type. Distributed parameters will define limits within which the characteristics assigned to a specific individual, will lie. For example In Simulex 3.0 the Office Staff 'group body type' will define a group of people with body sizes representing people with shoulder widths of $0.5 \mathrm{~m} \pm 15 \%$, and with unimpeded walking speeds ranging from $0.8-1.7 \mathrm{~m} / \mathrm{s}$. Simulex 3.0 uses random distribution within the specified limits, but other types of distribution may be used in later versions, as more detailed information becomes available about different population types.

\section{MOVEMENT PARAMETERS}

The following comments describe the main activities that occur within a modelled evacuation:

- RESPONSE TIME TO ALARM. Simulex 3.0 allows the user to specify a response time for the occupants. In Figure 3, the movement of the occupants in a defined area of the building will be delayed by an amount which lies randomly between the limits of $10-20$ seconds response time. This is only an example and it is intended that a later version of Simulex will incorporate a function which will assess certain factors and estimate the time taken for an occupant to assess his/her own response to an alarm with the user specifying certain conditions, instead of finite time limits. Data is currently being collected both at Edinburgh University and at Lund University in order to enable Simulex to assess the response of building occupants depending on different types of alarm, the familiarity of the occupants with the buildings, and a range of other factors. The proposed 'occupant response' model may also include some of the ideas discussed by Proulx \& Sime [5], and Proulx \& Hadjisophocleous [6]. The current use of specific time delays will be useful when assessing multi-storey buildings with phased evacuation where occupants on different floors are informed of the need to evacuate at different times.

- GEOMETRIC PARAMETERS FOR EACH PERSON include x,y co-ordinate position (defined with an accuracy of greater than $1 \mathrm{~mm}$ ), orientation (degrees) and body size \& type, based on the 3-circle body form described by Thompson \& Marchant [8] .

- ROUTE-FINDING: The optimal direction to an exit is calculated by using the previously defined distance map. For a given co-ordinate position in the building space, the surrounding distance map contours can be 'read' to yield an angle of travel such that the person travels at right angles to the contours of distance-to-exit. A sample distance map is illustrated in Figure 2. It is important to note that in this version of Simulex, the occupant does not necessarily head towards the nearest exit, but instead will use directions gained from the analysis of the chosen distance map(s), stored in the computer memory. 
- WALKING VELOCITY: Normal, unimpeded walking velocities are distributed among the population before the start of an evacuation, in accordance with parameters specified by the user. During an evacuation, the forward walking speed of each person, when obstructed by another person, is calculated by relating the inter-person distance (defined by Thompson \& Marchant - [8]) to a reduced walking velocity (Figure 2). Walking velocities are further reduced on staircases, by approximately $50 \%$, based on data obtained by Frantzich [3].

'BEST FIT' GRAPH OF VELOCITY AGAINST INTER-PERSON DISTANCE

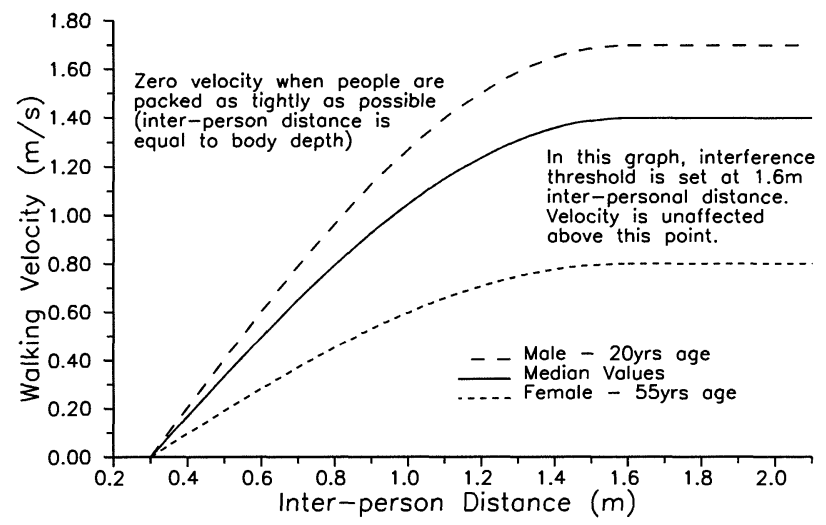

Figure 4. Best-Fit Graph of Velocity against Inter-Person Distance.

The use of this graph within the movement algorithms of Simulex 3.0 is important to ensure that Simulex produces realistic pedestrian flow rates, similar to those observed by Hankin \& Wright [4], and used in the BSI Code for Office Buildings [2]. It is based on data collected from the analysis of video footage of human movement described by Thompson [10]. Tests described by Thompson \& Marchant [9] have been carried out to analyse the effect of this graph shape on the flow rate achieved by groups of people through exit openings.

- RATES OF BODY TWIST: The rate at which individual people can twist or turn is limited in order that realistic crowd flow rates can be recreated by Simulex. The value used in for an able-bodied person is 100 degrees per second. This data is based upon figures produced by Rickets [10]. The rate of individual body twist has a significant effect on the overall flow rate of groups of people as they move through exit openings (Thompson \& Marchant - [9]). 
- OVERTAKING: Algorithms to assess alternative directions of overtaking for an obstructed person are integral to the movement functions within Simulex. Initially, the modelled person 'looks forward' in the optimal direction to an exit. If movement in this direction might be significantly impeded by the physical presence of other people, then alternative directions are assessed which allow the obstructed person to negotiate a path around the most immediate obstructing person. Collision-predictions based on the directions and speeds of movement of individuals are incorporated in order that contraflows of groups of people may be accurately modelled. The basic forms of overtaking are illustrated below in Figures 5 and 6.

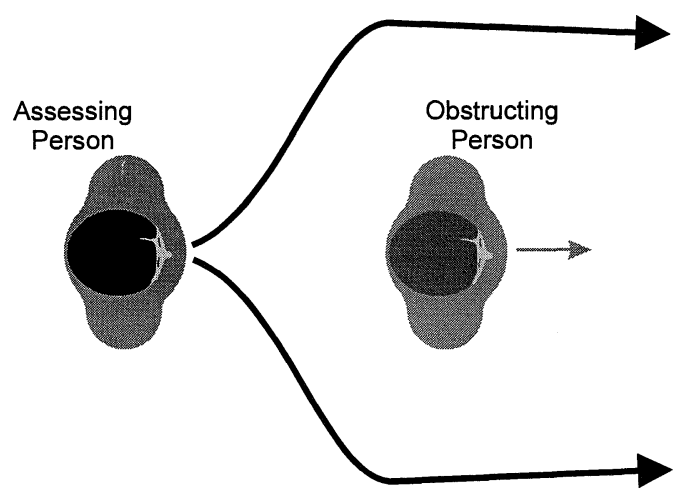

Figure 5. Overtaking Method for Obstructing Person Travelling in Similar Direction

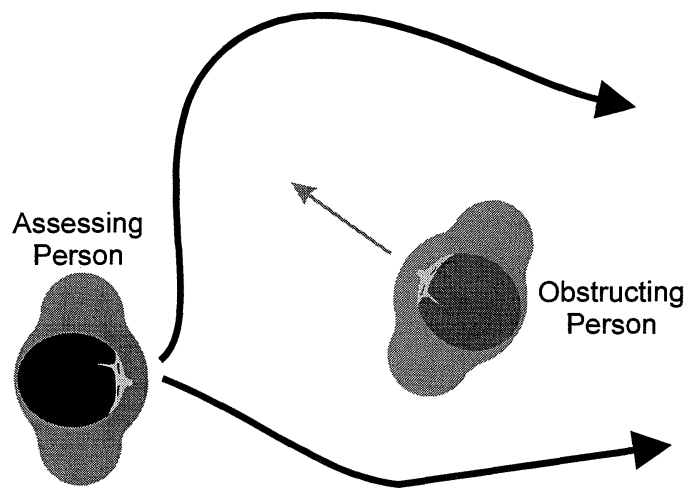

Figure 6. Overtaking for Obstructing Person Travelling in Opposing Direction 
The calculations for overtaking are based upon the assumption that the 'Assessing Person' will negotiate a path around the 'Obstructing Person' such that the bodies avoid contact by a minimum of $50 \mathrm{~mm}$. The value of $50 \mathrm{~mm}$ is a nominal value based on body-sway during forward movement (Bryan - [1]).

\section{THE SIMULATION PROCEDURE}

Simulex updates and recalculates the motion of each modelled person, using the movement parameters described in section 6 , at every time-step of 0.1 seconds.

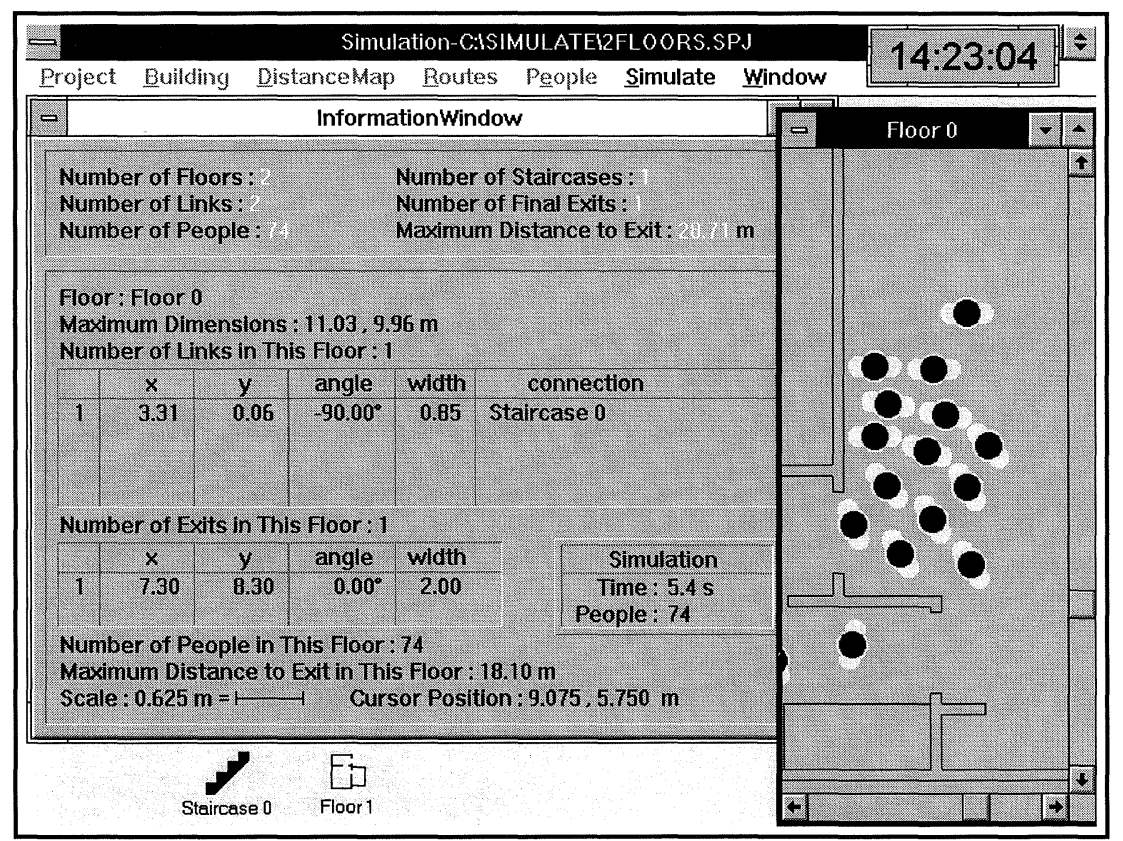

Figure 7. Sample Screen Display of a Group of Modelled People Walking Through a Doorway.

A number of facilities are available to the user during an evacuation. Whenever the user selects a particular staircase or floor plan as the current viewing window, then the 'Information Window' shown in Figure 7 will display the current information about that part of the building. The information includes the overall simulation time, the number of people on 
the floor/staircase and also detailed information about the number of exits and links which are directly associated with that part of the building.

The occurrence of localised crowding and queuing in Simulex 3.0 is a result of the use of the graph illustrated in Figure 4 to determine the walking speed of each individual at each timestep. The behaviour of the modelled people as they slow down and stop when they reach a congested area appears to be reasonably realistic. However, when people are in very close proximity at narrow door openings $(0.65-0.8 \mathrm{~m})$, the bodies can ' $\mathrm{jam}$ ' and halt the pedestrian flow through the opening. The authors believe that although this jamming is to be expected under certain conditions, it occurs too frequently when the lateral movement of individuals is only governed by the overtaking algorithms. As a result, when two bodies are touching the 'Assessing Person' needs to be able to make the decision to rotate his/her body and shuffle sideways through the narrow space available. The algorithms for this form of movement are currently under construction.

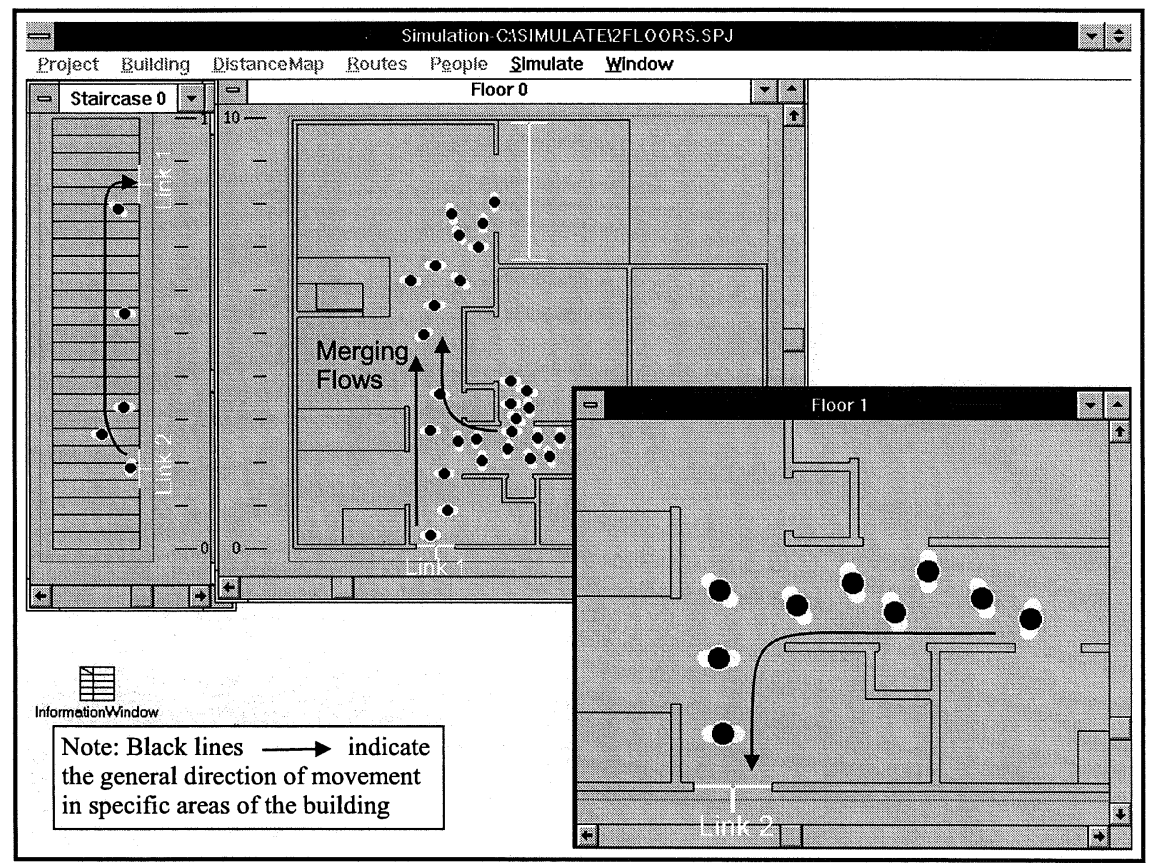

Figure 8. Sample Screen Display (with notation) of an Evacuation in a simple 3-storey Building.

Simulex can be used to recreate specific features of crowd movement which are observed in 'real-life'. Figure 8 illustrates the movement of different groups of people, that results in 'merging flow' In this evacuation. people were initially present in both floors of a building. As 
the evacuation progressed, people walked from 'Floor 1', into the staircase via 'Link 2', down the staircase and into 'Floor 0' via 'Link 1'. The people from the upper floor then meet the people on the lower floor in the main corridor. A 'merging flow' is set up when the different groups meet and head towards a common goal (the I-shaped line of exit). The overall speeds of movement of both groups are reduced by this merging-flow behaviour which produces a localised increase in crowd density.

Due to the complex nature of the directional assessment and movement algorithms, an evacuation cannot be modelled and displayed at real-time, but instead the calculations are executed and the motion of each person is recorded onto hard disk for later real-time playback and viewing.

\section{CONCLUSIONS AND DEVELOPMENT}

It is possible to model the evacuation of very large mixed-ability populations through buildings which possess spaces which are very geometrically complex. Simulex has already been used to model the evacuation of thousands of people from a proposed department store, with a large, complex, floor plan (Thompson \& Marchant - [9]). The software has been developed to import CAD files, accommodate multi-storey buildings, and define a number of different final exits. These developments have been carried out in order that Simulex may become a useful design tool for fire safety engineers.

The main outcomes of the development of this version of the software has been to increase the complexity of the both the building volume and the population characteristics; to improve the movement functions (overtaking and collision avoidance); and to create exit choices by the use of multiple distance maps in memory. In addition, the use of the Windows software architecture allows much larger distance maps to be formed and stored, and as a result even the largest of buildings can be modelled by Simulex 3.0.

The future development of Simulex will concentrate on a number of different areas. The capacity of the software to model the response of occupants to stimuli such as alarms or public address systems needs to be greatly improved if more realistic simulation of human behaviour is to be achieved. As yet, Simulex makes no attempt to model the effects that a visible and toxic hazard may have on the occupants. The introduction of output files from fire and smoke models into Simulex would enable a whole range of new parameters to be assessed, and the algorithms to handle these data files will need to be written. Again the complexity of the functions which process psychological input for each individual need to be improved.

The version of Simulex described in this paper is already a useful research and design tool. It allows the user to assess how the geometry of a large, complex building affects the evacuation of the occupants. The software yields information about the behaviour of the occupants in terms of the evacuation time for each floor of a building and where congestion, merging flows and queues occur. The first assessment of a building design may consist of two simulated evacuations: one where all occupants use the nearest exits, and another where only those exits that the occupants are familiar with, are used. 


\section{REFERENCES}

1. Bryan, J.L., Concepts of Egress Design, Section 7, Chap.3 in "Firesafety in Building Design and Construction", Wiley Publications, London, 1985.

2. BSI - British Standards Institution (1983), BS 5588: Part 3: 1983, Fire precautions and the design and construction of buildings: Part 3: Code of Practice for Office Buildings, London.

3. Frantzich, H., Study of Movement on Stairs During Evacuation using Video Analysing Techniques, Publication no. ISRN LUTVDG/TVBB_-3079--SE, Department of Fire Safety Engineering, University of Lund, Sweden, 1996.

4. Hankin, B.D. and Wright, R.A., Passenger Flows in Subways, Operational Research Quarterly, Vol. 9, pp 81-88, 1958.

5. Proulx, G. \& Sime, J.D., To Prevent Panic in an Underground Emergency: Why not tell people the truth ?, Proc. of the 3rd Int. Symp. of Fire Safety Science, University of Edinburgh, July 8-12 ,1991, Elsevier Science Publishers, London, 1991.

6. Proulx, G., \& Hadjisophocleous, G., Occupant Response Model: A Sub-Model for the NRCC Risk-Cost Assessment Model, in Proc. of the 4th Int.Symp. of Fire Safety Science, Ottawa Congress Centre, June 13- 17, 1994, Elsevier Science Publishers, London, 1994.

7. Ricketts, G., 1994, An Investigation into the Density and Flow of People in Public Spaces by Image Analysis, Hons. dissertation., Dept. of Civil Engineering and Building Science, University of Edinburgh.

8. Thompson, P.A. \& Marchant, E.W., A Computer Model for the Evacuation of Large Building Populations, Fire Safety Journal 24, 131-148, 1995.

9. Thompson, P.A. and Marchant, E.W., Testing and Application of the Computer Model 'Simulex', Fire Safety Journal 24, pp 149-166, 1995.

10. Thompson, P.A., Developing New Techniques for Modelling Crowd Movement, $\mathrm{PhD}$ Thesis, University of Edinburgh, Edinburgh, 1994. 